\title{
Prognostic analysis of patients with locally advanced nasopharyngeal carcinoma following intensity modulated radiation therapy
}

\author{
YAJIE ZHAO $^{1}$, LIN SHEN ${ }^{1}$, XINQIONG HUANG ${ }^{1}$, YUXIANG HE $^{1}, \mathrm{JUN} \mathrm{FU}^{1}$, \\ YUJIE QIAN $^{1}$, SHAN LI $^{1}$, NA ZHAO ${ }^{2}$ and LIANGFANG SHEN ${ }^{1}$ \\ ${ }^{1}$ Department of Oncology, Xiangya Hospital, Central South University, Changsha, Hunan 410008; \\ ${ }^{2}$ Department of Hospital Management Consulting Center, National Institute of Hospital Administration, \\ Beijing 100083, P.R. China
}

Received May 3, 2016; Accepted June 2, 2017

DOI: $10.3892 / \mathrm{ol} .2018 .7850$

\begin{abstract}
The present study retrospectively analyzed the prognostic factors of 135 patients with locally advanced nasopharyngeal carcinoma (NPC) who received intensity modulated radiation therapy between August 2008 and January 2012 at Xiangya Hospital of Central South University. Patients were staged from III-IVA according to the 7th American Joint Committee on Cancer staging system. Using Statistical Analysis System 9.3 software, the present study demonstrated that, among these 135 patients, the 5-year overall survival, the 5-year local relapse-free survival, and the 5-year disease metastasis-free survival were 84,82 , and $78 \%$, respectively. Multivariate Cox regression analysis identified that targeted treatment [hazard ratio (95\% confidence interval), 2.642 (1.001, 6.972); $\mathrm{P}=0.0497$ ] served as an independent negative prognostic factor in locally advanced NPC. The results of immunostaining revealed that the staining intensity of the radiation-resistant group was increased compared with that of the radiation-sensitive group. These results demonstrate that a high expression of EGFR may be associated with radiation resistance, and targeted treatment may not be effective in patients with locally advanced nasopharyngeal carcinoma with low expression of EGFR.
\end{abstract}

\section{Introduction}

Nasopharyngeal carcinoma (NPC) is a head and neck malignant tumor that is common to multiple provinces in southeastern China and Asia, and northern Africa (1). Radiotherapy

Correspondence to: Dr Liangfang Shen, Department of Oncology, Xiangya Hospital, Central South University, 87 Xiangya Road, Changsha, Hunan 410008, P.R. China

E-mail:wzyj7177@126.com

Key words: nasopharyngeal carcinoma, intensity modulated radiation therapy, prognosis factors, immunohistochemistry targeting the nasopharyngeal tumor and draining lymph node echelons remains a common treatment for NPC $(2,3)$. Although early stage NPC may be effectively treated by radiotherapy alone, treatments of regional advanced NPC (Union for International Cancer Control Stage III/IV) require improvements; the incidence rate of a local residual tumor following radical radiotherapy is $7.0-13.0 \%$ and the local recurrent rate ranges from 16.8 to $23.0 \%$ (4-8). To reduce the rate of local recurrence and distant metastasis, combination treatments, including chemoradiotherapy and molecular targeted therapy are particularly important for cases of locally advanced head and neck squamous-cell carcinoma (HNSCC) (9). Potential therapeutic targets for HNSCC, including epidermal growth factor receptor (EGFR), have been researched over the past decade. High EGFR expression is associated with poor prognosis in head and neck squamous-cell carcinoma and with resistance to radiotherapy $(10,11)$. A previous study demonstrated that nimotuzumab combined with radiotherapy or chemotherapy was associated with improved locoregional tumor control and survival compared with standard chemoradiotherapy in patients with locally advanced HNSCC (12). Cetuximab has been used with radiotherapy or in combination with platinum 5-fluorouracil in advanced or recurrent patients with HNSCC (13-16). However, the use of molecular targeted drugs has been hindered: The molecular targeted therapy causes skin toxicity, and the rates of treatment sensitivity require improvement. In addition, the effect of the drugs when combined with chemotherapy or radiotherapy remains incompletely understood. Pfister et al (12) revealed that combining cetuximab with radiotherapy or chemoradiotherapy resulted in drug-associated toxicities in HNSCC; the early trial was subsequently suspended. Numerous other anti-EGFR agents are currently being assessed in phases II and III clinical trials in different HNSCC therapeutic settings (17). None of these molecule-targeting drugs have yet been approved by the Food and Drug Administration for use in patients with HNSCC due to the limited improvement on survival with which they are associated.

The present study assessed which factors influenced the survival of 135 patients with locally advanced NPC following 
radiotherapy. The present study revealed that targeted treatment functions were an independent negative prognostic factor in patients with locally advanced NPC.

\section{Materials and methods}

Patient characteristics. The population of the present study comprised 135 patients (99 males, 36 females; $76 \geq 45$ years, $59<45$ years) with locally advanced NPC who received radical radiotherapy in the Department of Radiation Oncology, Xiangya Hospital, Central South University (Changsha, China) between August 2008 and January 2012. The inclusion criteria were as follows: i) Patients exhibited pathologically proved poorly differentiated squamous-cell carcinoma; ii) patients were staged in III-IVA according to the 7th American Joint Committee on Cancer (AJCC) staging system (18) with no evidence of distant metastasis at the time of diagnosis; and iii) patients did not receive any other anticancer treatments prior to primary radiotherapy or undergo an operation following radiotherapy. The duration of overall survival was calculated from the date of radiotherapy completion to the date of mortality in the patient or the date of the last follow-up. The duration of local relapse-free survival was calculated from the date of radiotherapy completion to the date of tumor local recurrence. The duration of disease metastasis-free survival was calculated from the date of radiotherapy completion to the date of tumor distant metastasis $(19,20)$.

Intensity modulated radiation therapy. In accordance with the International Commission on Radiation Units and Measurements (ICRU) reports nos. 50 and 62 (21,22), the primary tumor was named GTVnx, the positive lymph nodes were named GTVnd, and the retropharyngeal lymph nodes were included under the name GTVnx. Clinical target volume 1 (CTV1) included a 5-10 $\mathrm{mm}$ extension around the GTVnx and other high-risk regions, including the parapharyngeal space, the inferior part of sphenoid sinus, the posterior $1 / 3$ of the nasal cavity, the posterior $1 / 3$ of the maxillary sinus, the skull base, the clivus, the oval foramen, the lacerated foramen, and high-risk lymphatic drainage areas, including the retropharyngeal lymph nodes, and levels II, III, and Va of the upper cervical lymph nodes. CTV2 included the low-risk lymphatic drainage levels IV and $\mathrm{Vb}$. The corresponding planning target volume (PTV) included a $3 \mathrm{~mm}$ margin around the CTV. A total dose of $62.72-80.64 \mathrm{~Gy}$ in 33 fractions to the PGTVnx (GTVnx + $3 \mathrm{~mm}$ margin), 69.96-72.6 Gy in 33 fractions to the PGTVnd (GTVnd $+3 \mathrm{~mm}$ margin), 59.4-64.0 Gy in 33 fractions to the PTV1 and 50.0-54.0 Gy in 28 fractions to the PTV2 were prescribed. All patients were treated with 1 fraction daily, 5 days/week, for 5-6 weeks $(23,24)$. Dose limits for the critical tissue structures and the plan evaluation were designed according to the Radiation Therapy Oncology Group 0225 (25). The patients were re-examined by magnetic resonance imaging (MRI) following either the completion of radiotherapy or the radiation dose reaching $\sim 70 \mathrm{~Gy}$ (26). All patients were exposed to $6 \mathrm{MV}$ beams and 9 fixed-gantry $(0,40$, $80,120,160,200,240,280$ and $320^{\circ}$ ) angles were designated. Plans were all normalized so that $95 \%$ of the target received $\geq 100 \%$ prescription dose.
Chemotherapy. To inhibit NPC progression during treatment planning, 131 of the patients received a platinum-based chemotherapy regimen. The chemotherapy regimens included Taxol $\left(120 \mathrm{mg} / \mathrm{m}^{2}\right.$ on day $1+80 \mathrm{mg} / \mathrm{m}^{2}$ Cislatin/Nedaplatinon day 2; for 3 weeks/cycle), Gemcitaine $\left(1 \mathrm{~g} / \mathrm{m}^{2}\right.$ on day 1 and $8+80 \mathrm{mg} / \mathrm{m}^{2}$ Cislatin/Nedaplatinon day 1 , for 3 weeks/cycle) or $5-\mathrm{Fu}\left[4 \mathrm{~g} / \mathrm{m}^{2}\right.$ continuous infusion $(\mathrm{CI})>96 \mathrm{~h}+80 \mathrm{mg} / \mathrm{m}^{2}$ Cislatin/Nedaplatinon day 1, for 3 weeks/cycle], for 2-6 cycles. The remaining 4 patients did not undergo chemotherapy since they were unwilling or unable. Neoadjuvant chemotherapy was administered when the waiting time for radiotherapy $>1$ week or to decrease the size of large tumors. Following the completion of radiotherapy, adjuvant chemotherapy was administered to the patients at stage $\mathrm{N} 2 / \mathrm{N} 3$ and those with existing residual disease, as detected using MRI or physical examination.

Sensitization treatment. Intravenously, 76 patients received $750 \mathrm{mg}$ sodium glycididazole $/ \mathrm{m}^{2} /$ fraction, 3-5 fractions/week for 5-6 weeks, within a 1 or $2 \mathrm{~h}$ window, to ensure that the drug remained active during administration.

Target treatment. A monoclonal antibody treatment of EGFR was administered to 20 of the patients who were able to purchase the drug. Of these, 16 received 100-200 mg nimotuzumab/week for 6-8 weeks, while 4 received $400 \mathrm{mg}$ cetuximab $/ \mathrm{m}^{2}$ as an initial dose and $250 \mathrm{mg}$ cetuximab $/ \mathrm{m}^{2} /$ week for 6-8 weeks, the duration of treatment was determined by the patient response to the drug.

Immunohistochemical analysis. Biopsy specimens from the 20 patients with NPC who had been receiving targeted drugs were $10 \%$ formalin-fixed and paraffin-embedded. Samples were fixed in $10 \%$ formalin at room temperature for $48 \mathrm{~h}$. For the immunohistochemical detection of EGFR, $4 \mu \mathrm{m}$ tissue sections were deparaffinized in xylene and subsequently exposed to microwaves in a microwave oven $(750 \mathrm{~W})$ at $60-70^{\circ} \mathrm{C}$ for 15 min in EDTA buffer (pH 9.0, G1202). Following cooling for $30 \mathrm{~min}$ and washing in PBS, endogenous peroxidase was blocked with $3 \%$ hydrogen peroxide for $25 \mathrm{~min}$. Specimens were subsequently incubated at room temperature in a humidified box with PBS containing 10\% normal goat serum for $30 \mathrm{~min}$. Specimens were incubated overnight at $4^{\circ} \mathrm{C}$ with the anti-EGFR antibody (cat. no. bs-0165R, 1:200; BIOSS, Beijing, China). A horseradish peroxidase-labeled goat anti-mouse IgG secondary antibody (dilution 1:1,000, cat. no. K5007; Dako; Agilent Technologies, Inc., Santa Clara, CA, USA) was then incubated with the tissue for $30 \mathrm{~min}$ at $37^{\circ} \mathrm{C}$. Immunostaining was detected using the ChemMate kit (cat. no. K5007, ready-to-use; Dako; Agilent Technologies, Inc.) with 3,3'-diaminobenzidine as the chromogen. For the negative control, the primary antibody was replaced with non-immune isotypic antibodies. The protocol was repeated in triplicate.

Evaluation of staining. The stained sections were viewed separately by two pathologists (Department of Pathology, Xiangya Hospital, Central South University, Changsha, China) who were blinded to the clinical or clinicopathological status of the cases. EGFR expression was evaluated by scanning the entire tissue specimen under low power magnification $(x 40)$ and subsequently confirmed under high power magnification 
(x400). The result (positive or negative) was diagnosed through stereological cell counts. The absence of positive cells resulted in a negative diagnosis. When $<25 \%$ of the cells were positive the diagnosis was weakly positive. A moderately positive diagnosis was made when the proportion of positive cells was $25-50 \%$. A strongly positive diagnosis was made when $>50 \%$ of the cells were positive. According to this method of assessment, staining scores of negative and weakly positive were regarded as representing tumors with decreased EGFR expression, while staining scores of moderately and strongly positive were regarded as representing tumors with increased EGFR expression.

Follow-up. The follow-up methods included direct telephone calls to the patients or their families, or hospital visits for the patients. Follow-up was measured from the first day of treatment to the last follow-up date or the date of patient mortality. Following radiotherapy, follow-up examinations were conducted once every 3 months in the first 2 years, once every 6 months in years 2 to 5 , and once annually following this. MRI of the nasopharynx and neck region was performed once annually for patients with no residual tumors and once every 3-6 months for patients with residual tumors, as described previously (27). Recurrence was defined as regrowth of the tumor following disappearance for $\geq 1$ month. The follow-up began at August 2008 and ended in September 2015. The median follow-up for survivors was 48 months (range, 2-75 months). In addition, only 129 of the 135 patients were eligible for survival analysis due the loss of follow-up for 6 patients who were treated as mortalities. The follow-up rate was $95.6 \%$.

Statistical analysis. All statistical analysis was performed using Statistical Analysis System 9.3 software. Survival curves were calculated using Kaplan-Meier estimates and differences were compared using the log-rank test. Univariate and multivariate survival analysis was performed according to the Cox proportional hazards model. For all statistical tests, $\mathrm{P} \leq 0.05$ was considered to indicate a statistically significant difference.

\section{Results}

Clinical characteristics of patients. The clinical characteristics of 135 patients with locally advanced NPC were presented in Table I. Of these patients, 59 were $<45$ years and 76 were $\geq 45$ years; 19 exhibited AJCC (7th) stage III NPC and 116 exhibited AJCC (7th) stage IVA NPC. Histologically, all patients were classified as exhibiting poorly differentiated squamous-cell carcinoma according to the World Health Organization classification (28). Of the 135 patients enrolled in the present study, 20 received EGFR monoclonal antibody treatment and the remainder did not.

Only 129 of the 135 patients underwent survival analysis. In total, 4/129 patients developed local recurrence $(3.1 \%)$, 20/129 patients developed distant metastasis (15.5\%), and $1 / 129$ patients developed recurrence and distant metastasis $(0.8 \%)$. Mortality was recorded in $22 / 129$ patients $(17.1 \%)$; 17 of these mortality cases were due to tumor recurrence and metastasis, 3 were due to tumor-associated complications (nasopharyngeal hemorrhage, 2 cases; septic shock, 1 case),
Table I. Characteristics and Kaplan-Meier analysis of 135 patients with locally advanced nasopharyngeal carcinoma.

\begin{tabular}{|c|c|c|c|}
\hline Parameters & $\mathrm{n}$ & $\chi^{2}$ & P-value \\
\hline Sex & & 0.0704 & 0.7907 \\
\hline Male & 99 & & \\
\hline Female & 36 & & \\
\hline Age (years) & & 5.3076 & 0.0212 \\
\hline$<45$ & 59 & & \\
\hline$\geq 45$ & 76 & & \\
\hline AJCC (7th) stage & & 0.3251 & 0.5685 \\
\hline III & 19 & & \\
\hline IVA & 116 & & \\
\hline Chemotherapy (platinum-based) & & 0.2491 & 0.6177 \\
\hline Yes & 131 & & \\
\hline No & 4 & & \\
\hline Prescribed dose, Gy & & 0.8668 & 0.3518 \\
\hline$\leq 73.92$ & 114 & & \\
\hline$>73.92$ & 21 & & \\
\hline Targeted treatment & & 4.9193 & 0.0266 \\
\hline Yes & 20 & & \\
\hline No & 115 & & \\
\hline Sensitization treatment & & 0.5548 & 0.4564 \\
\hline Yes & 76 & & \\
\hline No & 59 & & \\
\hline
\end{tabular}

AJCC, American Joint Committee on Cancer; n, number of patients.

1 was due to malnutrition systemic failure and 1 was due to unknown causes.

Univariate and multivariate Coxregression analyses. Univariate analyses revealed that patient age $\left(\chi^{2}=5.3076\right.$, $\mathrm{P}=0.0212)$ and targeted treatment $\left(\chi^{2}=4.9193, \mathrm{P}=0.0266\right)$ were negative prognostic predictors of overall survival in patients with locally advanced NPC (Fig. 1A and B). Multivariate Cox regression analysis indicated that targeted treatment [hazard ratio (95\% confidence interval), 2.642 (1.001, 6.972); $\mathrm{P}=0.0497]$ was a negative prognostic predictor of overall survival in patients with locally advanced NPC (Table II).

Immunostaining analysis. To further evaluate the clinical significance of EGFR, the protein expression levels of EGFR in 20 paraffin-embedded NPC biopsy specimens from 20 patients with locally advanced NPC receiving targeted treatment were determined using immunohistochemical analysis. The present study defined patients for which tumor size had decreased by $<40 \%$ following radiation treatment as radiation-resistant, and those for which tumor size had decreased by $>60 \%$ as radiation-sensitive (29). Of the 20 patients, 2 belonged to the radiation-resistant group and revealed increased expression of EGFR compared with the radiation-sensitive group; tumor size decreased in these patients by 37.1 and $34.6 \%$ compared with initial size. The remaining 18 patients belonged to the radiation-sensitive group, with 17 demonstrating decreased 
Table II. Multivariate Cox regression analysis of 135 patients with locally advanced nasopharyngeal carcinoma.

\begin{tabular}{lccc}
\hline Variables & Subset & Hazard ratio (95\% CI) & P-value \\
\hline Age, years & $<45$ vs. $\geq 45$ & $2.836(0.932,8.631)$ & 0.0664 \\
Targeted treatment & Yes vs. No & $2.642(1.001,6.972)$ & 0.0497 \\
Prescribed dose, Gy & $\leq 73.92$ vs. $>73.92$ & $0.528(0.117,2.377)$ & 0.4050 \\
\hline
\end{tabular}

CI, confidence interval.

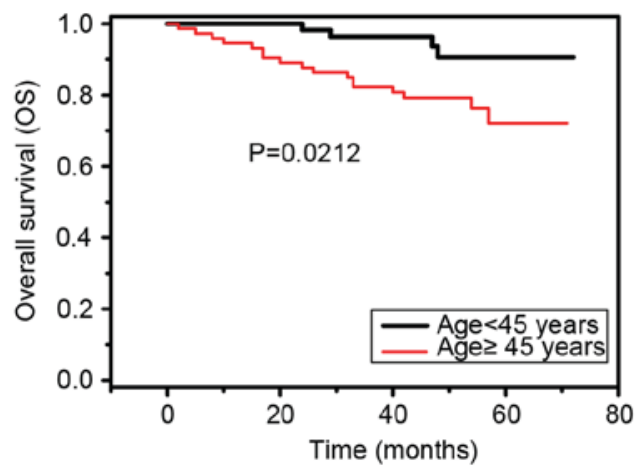

B

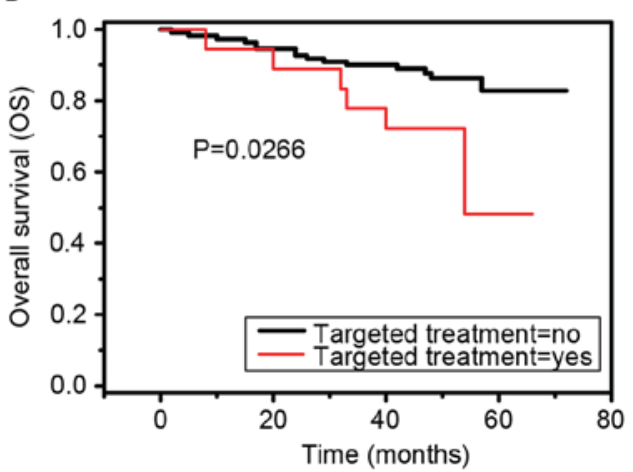

Figure 1. Kaplan-Meier survival analysis for various clinical factors. (A) Age of diagnosis in OS. (B) Targeted treatment in OS. OS, overall survival.

A

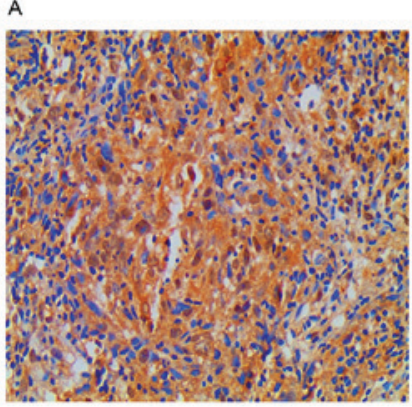

B

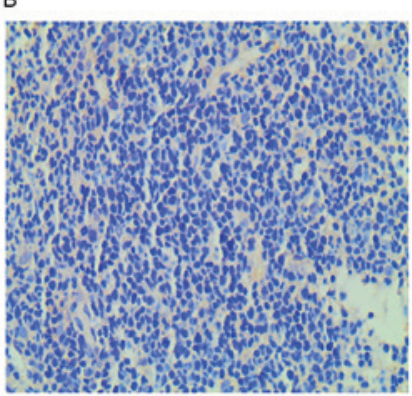

Figure 2. Representative examples of EGFR staining of tumors in the radiation-resistant and radiation-sensitive groups (magnification, $x 400$ ). (A) Increased EGFR-positive staining intensity in the radiation-resistant group. (B) Decreased EGFR-positive staining in the radiation-sensitive group. Scale bar, $90 \mu \mathrm{M}$. EGFR, epidermal growth factor receptor.

expression of EGFR and 1 revealing increased expression of EGFR: Tumor size decreased by $73-100 \%$ in the radiation-sensitive group, compared with initial size. EGFR was located in the nasopharyngeal carcinoma cell cytoplasm. There was decreased EGFR expression in 17 of the 20 patients $(85 \%)$ and increased EGFR expression in the remaining 3 patients $(15 \%)$. The average staining intensity was increased in the radiation-resistant group compared with the radiation-sensitive group (Fig. 2A and B).

\section{Discussion}

EGFR-specific monoclonal antibodies, including cetuximab and nimotuzumab, have been researched in multiple types of cancer, including sarcoma (30), gliomas (31), and non small cell lung cancer (NSCLC) (32). These antibodies may inhibit the downstream growth-signaling pathway and may serve as a marker of tumorigenesis in NSCLC and glioma (33). However, few studies have assessed the use of targeted drugs in patients with NPC when combined with chemotherapy or radiation.

The present study enrolled 135 patients with locally advanced NPC, of which 20 received targeted treatment. The present study demonstrated that targeted treatment functions as an independent negative prognostic factor in patients with locally advanced NPC. Immunostaining analysis identified that the staining intensity of the radiation-resistant group was increased compared with that of the radiation-sensitive group. Although 1 patient in the radiation-sensitive group revealed increased expression of EGFR, the staining intensity of the tissue derived from the patient was relatively decreased compared with that from the patients of the radiation-resistant group. This result supported studies by Pan et al (34) and Ma et al (35), which demonstrated that EGFR overexpression was associated with radiation resistance in head and neck cancer, and prognostically associated with a decreased local control rate and survival. Multiple studies have revealed that $\sim 80 \%$ of primary NPC biopsies demonstrate increased expression of $\operatorname{EGFR}(36,37)$. In the present study, there were $2 / 20$ patients who received targeted treatment that belonged to radiation-resistant group, these patients exhibited EGFR over expression. A total of $18 / 20$ who received targeted treatment belonged to the radiation-sensitive group, only 1 patient in this group exhibited EGFR over expression. In the present study, only $15 \%$ of the patients demonstrated increased expression of EGFR.

No consensus has yet been reached regarding the effectiveness of an EGFR-targeting treatment in patients with NPC. 
Pfister et al (12) suggested that combining cetuximab and radiotherapy or chemotherapy may improve loco-regional tumor control and the survival rate compared with conventional chemoradiotherapy in locally advanced HNSCC. Cohen et al (17) revealed that cetuximab, which may act as a radiosensitizer, when combined with conventional chemoradiotherapy with cisplatin remained an ineffective treatment for patients with HNSCC. This result may differ from the present study due to EGFR mutation status. Although EGFR mutations are reported to have a decreased prevalence (0-1\%) in patients with NPC compared with other NPC-associated genes $(38,39)$, they may serve an important function in tumor development where they do occur; for example, the mitogen-activated protein kinase/extracellular signal-regulated kinase pathway is downstream of EGFR and may lead to cetuximab resistance in patients with NPC $(40,41)$.

Numerous drugs are in the early stages of development for HNSCC treatment, including novel anti-EGFR small-molecule tyrosine kinase inhibitors, EGFR antisense molecules, and multiple add-on therapies to radiation and chemotherapy intended to decrease resistance to anti-EGFR agents (42-45). In addition, numerous other anti-EGFR agents are currently being assessed in phases II and III clinical trials in different HNSCC therapeutic settings $(46,47)$. As the targeted treatment group only had $3 / 20$ patients with high expression of EGFR, the majority of the patients exhibited low expression of EGFR; consequently, those patients were not sensitive to cetuximab and nimotuzumab. Multiple studies have revealed that almost $80 \%$ of primary NPC biopsies indicate high expression of EGFR $(36,37)$. EGFR of $>25 \%$ was associated with a significantly poorer treatment outcome. The 5-year disease-specific survival, relapse-free survival, loco-regional relapse-free, and distant metastasis-free rates in patients with an EGFR of $>25 \%$ were $48,36,60$, and $55 \%$, respectively. The corresponding rates in patients with an EGFR of $<25 \%$ were $86,80,93$, and $86 \%$, respectively. The differences were all statistically significant, with the exception of distant metastasis. In multivariate analysis, EGFR extent was the only independent factor that predicted disease relapse, loco-regional failure, and mortality due to cancer (48). The present study hypothesized that treatment insensitivity was the primary reason for this unsatisfied curative effect. Collectively, the results obtained from these previously studies were similar to those presented in the present study. Results of the present study demonstrated that $<25 \%$ EGFR in patients with NPC is associated with reduced overall survival. The prognosis was not only concerned with the positive expression rate of EGFR; however, it also had an association with the amount of EGFR. The possible efficacy of molecular targeted therapy against EGFR is a promising treatment strategy, future studies examining the expression of EFGR prior to treatment in patients with advance-stage NPC are required. Of course, the number of subjects should not be discounted; only $2 / 20$ patients belonged to the radiation resistance group, and therefore the sample size was too small to perform meaningful statistical analysis independently. Validating the results of the present study requires further biopsy collections to assess the expression of EGFR in patients with NPC and whether EGFR expression is associated with radiation resistance. The limitations of the present study were as follows: On one hand, the volume of nasopharyngeal tumor tissue was small $\left(<0.5 \mathrm{~cm}^{3}\right)$, meaning that they were difficult to isolate under the nasopharyngeal microscope. Additionally, targeted treatment was not included as a part of the standard therapy: Only patients with local advanced NPC who could afford the treatment were able to select the targeted drugs. The present study suggested that treatment with cetuximab and nimotuzumab may be associated with the expression of EGFR. However, it may not be effective in patients with low expression of EGFR, despite this EGFR remains an attractive target for treating certain types of cancer.

\section{Acknowledgements}

The present study was supported by National Natural Science and Technology Major Foundation of China (grant no. SQ2017ZX090361); National Natural Science Foundation of China (grant nos. 81372792 and 81602683); Hunan Department of Scienceand Technology Foundation (grant nos. 2016SK2007 and 2015JJ4055).

\section{References}

1. Jemal A, Bray F, Center MM, Ferlay J, Ward E and Forman D: Global cancer statistics. CA Cancer J Clin 61: 69-90, 2011.

2. Wei WI and Sham JS: Nasopharyngeal carcinoma. Lancet 365: 2041-2054, 2005

3. Chang JT, See LC, Liao CT, Ng SH, Wang CH, Chen IH, Tsang NM, Tseng CK, Tang SG and Hong JH: Locally recurrent nasopharyngeal carcinoma. Radiother Oncol 54: 135-142, 2000.

4. Stoker SD, van Diessen JNA, de Boer JP, Karakullukcu B, Leemans CR and Tan IB: Current treatment options for local residual nasopharyngeal carcinoma. Curr Treat Options Oncol 14: 475-491, 2013.

5. Qiu S, Lin S, Tham IW, Pan J, Lu J and Lu JJ: Intensity-modulated radiation therapy in the salvage of locally recurrent nasopharyngeal carcinoma. Int J Radiat Oncol Biol Phys 83: 676-683, 2012.

6. Lee AW, Sze WM, Au JS, Leung SF, Leung TW, Chua DT, Zee BC, Law SC, Teo PM, Tung SY, et al: Treatment results for nasopharyngeal carcinoma in the modern era: The Hong Kong experience. Int J Radiat Oncol Biol Phys 61: 1107-1116, 2005.

7. Yeh SA, Tang Y, Lui CC, Huang YJ and Huang EY: Treatment outcomes and late complications of 849 patients with nasopharyngeal carcinoma treated with radiotherapy alone. Int J Radiat Oncol Biol Phys 62: 672-679, 2005.

8. Baujat B, Audry H, Bourhis J, Chan AT, Onat H, Chua DT, Kwong DL, Al-Sarraf M, Chi KH, Hareyama M, et al: Chemotherapy in locally advanced nasopharyngeal carcinoma: An individual patient data meta-analysis of eight randomized trials and 1753 patients. Int J Radiat Oncol Biol Phys 64: 47-56, 2006.

9. Vermorken JB and Specenier P: Optimal treatment for recurrent/metastatic head and neck cancer. Ann Oncol 21 (Suppl 7): vii252-vii261, 2010.

10. Bianco C, Tortora G, Bianco R, Caputo R, Veneziani BM, Caputo R, Damiano V, Troiani T, Fontanini G, Raben D, et al: Enhancement of antitumor activity of ionizing radiation by combined treatment with the selective epidermal growth factor receptor-tyrosine kinase inhibitor ZD1839 (Iressa). Clin Cancer Res 8: 3250-3258, 2002.

11. Ai Z, Wang J, Wang Y, Lu L, Tong J and Teng Y: Overexpressed epidermal growth factor receptor (EGFR)-induced progestin insensitivity in human endometrial carcinoma cells by the EGFR/mitogen-activated protein kinase signaling pathway. Cancer 116: 3603-3613, 2010.

12. Pfister DG, Su YB, Kraus DH, Wolden SL, Lis E, Aliff TB, Zahalsky AJ, Lake S, Needle MN and Shaha AR: Concurrent cetuximab, cisplatin, and concomitant boost radiotherapy for locoregionally advanced squamous cell head and neck cancer: A pilot phase II study of a new combined-modality paradigm. J Clin Oncol 24: 1072-1078, 2006. 
13. Du Y, Peyser ND and Grandis JR: Integration of molecular targeted therapy with radiation in head and neck cancer. Pharmacol Ther 142: 88-98, 2014.

14. Bonner JA, Harari PM, Giralt J, Azarnia N, Shin DM, Cohen RB, Jones CU, Sur R, Raben D, Jassem J, et al: Radiotherapy plus cetuximab for squamous-cell carcinoma of the head and neck. N Engl J Med 354: 567-578, 2006.

15. Bonner JA, Harari PM, Giralt J, Cohen RB, Jones CU, Sur RK, Raben D, Baselga J, Spencer SA, Zhu J, et al: Radiotherapy plus cetuximab for locoregionally advanced head and neck cancer: 5 -year survival data from a phase 3 randomised trial, and relation between cetuximab-induced rash andsurvival. Lancet Oncol 11: 21-28, 2010.

16. Vermorken JB, Mesia R, Rivera F, Remenar E, Kawecki A, Rottey S, Erfan J, Zabolotnyy D, Kienzer HR, Cupissol D, et al: Platinum-based chemotherapy plus cetuximab in head and neck cancer. N Engl J Med 359:1116-1127, 2008.

17. Cohen RB: Current challenges and clinical investigations of epidermal growth factor receptor (EGFR) and ErbB family-targeted agents in the treatmentof head and neck squamous cell carcinoma (HNSCC). Cancer Treat Rev 40: 567-577, 2014.

18. Edge SB and. Compton CC: The American joint committee on cancer: The 7th edition of the AJCC cancer staging manual and the future of TNM. Ann Surg Oncol 17: 1471-1474, 2010

19. Huang XQ, Chen X, Xie XX, Zhou Q, Li K, Li S, Shen LF and $\mathrm{Su}$ J: Co-expression of CD147 and GLUT-1 indicates radiation resistance and poor prognosis in cervical squamous cell carcinoma. Int J Clin Exp Pathol 7: 1651-1666, 2014.

20. Zhao Y, Shen L, Chen X, Qian Y, Zhou Q, Wang Y, Li K, Liu M, Zhang $S$ and Huang X: High expression of PKM2 as a poor prognosis indicatoris associated with radiation resistance in cervical cancer. Histol Histopathol 30: 1313-1320, 2015

21. International Commission on Radiation Units and Measurements (ICRU), ICRU Report: Prescribing, recording, and reporting photon beam therapy. Vol 50. ICRU, Bethesda, MD, 1993.

22. International Commission on Radiation Units and Measurements (ICRU), ICRU Report: Prescribing, recording, and reporting photon beam therapy. Vol 62. ICRU, Bethesda, MD, 1999 (supplement to ICRU report 50).

23. Zhao Y, Shen L, Huang X, Jing D, Huang D, Fu J, Li Z, Zhang G and Shen L: High expression of Ki-67 acts a poor prognosis indicator in locally advanced nasopharyngeal carcinoma. Biochem Biophys Res Commun 494: 390-396, 2017.

24. Zhou Q, He Y, Zhao Y, Wang Y, Kuang W and Shen L: A study of 358 cases of locally advanced nasopharyngeal carcinoma receiving intensity-modulated radiation therapy: Improving the seventh edition of the American joint committee on cancer T-staging system. Biomed Res Int 2017: 1419676, 2017.

25. Radiation Therapy Oncology Group: A Phase II Study of Intensity Modulated Radiation Therapy (IMRT) ${ }^{+/-}$Chemotherapy for Nasopharyngeal Cancer. RTOG protocol 0225, 2014. (www. rtog.org).

26. He Y, Zhou Q, Shen L, Zhao Y, Lei M, Wei R, Shen L and Cao S A retrospective study of the prognostic value of MRI-derived residual tumors at the end ofintensity-modulated radiotherapy in 358 patients with locally-advanced nasopharyngeal carcinoma. Radiat Oncol 10: 89, 2015.

27. Liu X, Liu LZ, Mao YP, Chen L, Tang LL, Zhou GQ, Sun Y, Yue D, Lin AH, Li L and Ma J: Prognostic value ofmagnetic resonance imaging-detected cranial nerve invasion in nasopharyngeal carcinoma. Br J Cancer 110: 1465-1471, 2014.

28. Hsu HC, Chen CL, Hsu MM, Lynn TC, Tu SM and Huang SC: Pathology of nasopharyngeal carcinoma. Proposal of a new histologic classification correlated with prognosis. Cancer 59: 945-951, 1987.

29. Yang S, Chen J, Guo Y, Lin H, Zhang Z, Feng G, Hao Y, Cheng J, Liang $\mathrm{P}$, Chen $\mathrm{K}$, et al: Identification of prognostic biomarkers for response to radiotherapy by DNA microarray in nasopharyngeal carcinoma patients. Int J Oncol 40: 1590-1600, 2012.

30. Little SE, Bax DA, Rodriguez-Pinilla M, Natrajan R, Messahel B, Pritchard-Jones K, Vujanic GM, Reis-Filho JS and Jones C: Multifaceted dysregulation of the epidermal growth factor receptor pathway in clear cell sarcoma of the kidney. Clin Cancer Res 13: 4360-4364, 2007.

31. Ekstrand AJ, James CD, Cavenee WK, Seliger B, Pettersson RF and Collins VP: Genes for epidermal growth factor receptor, transforming growth factor alpha, and epidermal growth factor and their expression in human gliomas in vivo. Cancer Res 51: 2164-2172, 1991.
32. Qi DL, Cui Y, Wang QS, Huang CB, Xu J, Yang YZ, Xin L, Tian Y and Qi XA: A clinical trail on docetaxel and carboplatin therapy with or without nimotuzumab for the treatment of advanced nonsmall cell lung cancer. J Cancer Res Ther 11 (Suppl 1): C32-C37, 2015.

33. Shin DM, Ro JY, Hong WK, Hittelman WN: Dysregulation of epidermal growth factor receptor expression in premalignant lesions during head and neck tumorigenesis. Cancer Res 54 3153-3159, 1994

34. Pan J, Tang T, Xu L, Lu JJ, Lin S, Qiu S, Chen G and K Tham IW: Prognostic significance of expression of cyclooxygenase-2, vascular endothelial growth factor, and epidermal growth factor receptor in nasopharyngeal carcinoma. Head Neck 35: $1238-1247,2013$

35. Ma BB, Poon TC, To KF, Zee B, Mo FK, Chan CM, Ho S, Teo PM, Johnson PJ and Chan AC: Prognostic significance of tumor angiogenesis, Ki67, p53 oncoprotein, epidermal growth factor receptor and HER2 receptor protein expression in undifferentiated nasopharyngeal carcinoma-a prospective study. Head Neck 25: 864-872, 2003 .

36. Soo R, Putti T, Tao Q, Goh BC, Lee KH, Kwok-Seng L, Tan L and Hsieh WS: Overexpression of cyclooxygenase-2 in nasopharyngeal carcinoma and association with epidermal growth factor receptor expression. Arch Otolaryngol Head Neck Surg 131:147-152, 2005.

37. Pan J, Kong L, Lin S, Chen G, Chen Q and Lu JJ: The clinical significance of coexpression of cyclooxygenases-2, vascular endothelial growth factors, and epidermal growth factor receptor in nasopharyngeal carcinoma. Laryngoscope 118: 1970-1975, 2008.

38. Lee SC, Lim SG, Soo R, Hsieh WS, Guo JY, Putti T, Tao Q, Soong R and Goh BC: Lack of somatic mutations in EGFR tyrosine kinase domain in hepatocellular and nasopharyngeal carcinoma. Pharmacogenet Genomics 16: 73-74, 2006.

39. Zhang ZC, Fu S, Wang F, Wang HY, Zeng YX and Shao JY: Oncogene mutational profile in nasopharyngeal carcinoma. Onco Targets Ther 7: 457-467, 2014.

40. Zuo Q, Shi M, Chen J and Liao W: The Ras signaling pathway mediates cetuximab resistance in nasopharyngeal carcinoma. Biomed Pharmacother 65: 168-174, 2011.

41. Fernández-Medarde A and Santos E: Ras in cancer and developmental diseases. Genes Cancer 2: 344-358, 2011

42. Bozec A, Etienne-Grimaldi MC, Fischel JL, Sudaka A, Toussan N, Formento P and Milano G: The mTOR-targeting drug temsirolimus enhances the growth-inhibiting effects of the cetuximabbevacizumab-irradiation combination on head and neck cancer xenografts. Oral Oncol 47: 340-344, 2011

43. Chung $\mathrm{CH}$, Wang $\mathrm{H}$, Tsottles N, Gourin CG, Agrawal N, Molinolo A, Gutkind S, Forastiere AA, Marur S: A phase I study of everolimus in combination with cetuximab and cisplatin as first-line therapy in recurrent and metastatic $(\mathrm{R} / \mathrm{M})$ head and neck squamous cell carcinoma (HNSCC). J Clin Oncol 30 (Suppl), 2012.

44. Argiris A, Ohr J, Kubicek GJ, Duvvuri U, Heron DE, Kotsakis AP, Spencer C, Kim S, Grandis JR, Johnson JT, et al: Phase II randomized trial of radiotherapy (RT), cetuximab (E), and pemetrexed (Pem) with or without bevacizumab (B) in locally advanced squamous cell carcinoma of the head and neck (SCCHN). J Clin Oncol 31 (Suppl): S6043-S6043, 2013.

45. Xu M, Mizoguchi I, Morishima N, Chiba Y, Mizuguchi J and Yoshimoto T: Regulation of antitumor immune responses by the IL-12 family cytokines, IL-12, IL-23, and IL-27. Clin Dev Immunol 2010: 832454, 2010.

46. Yoo DS, Kirkpatrick JP, Craciunescu O, Broadwater G, Peterson BL, Carroll MD, Clough R, MacFall JR, Hoang J, Scher RL, et al: Prospective trial of synchronous bevacizumab, erlotinib, and concurrent chemoradiation in locally advanced head and neck cancer. Clin Cancer Res 18: 1404-1414, 2012.

47. Argiris A, Kotsakis AP, Hoang T, Worden FP, Savvides P, Gibson MK, Gyanchandani R, Blumenschein GR Jr, Chen HX, Grandis JR, et al: Cetuximab and bevacizumab: Preclinical data and phase II trial in recurrent or metastatic squamous cell carcinoma of the head and neck. Ann Oncol 24: 220-225, 2013.

48. Liu MT, Chen MK, Huang CC and Huang CY: Prognostic value of molecular markers and implication for molecular targeted therapies in nasopharyngeal carcinoma: An update in an era of new targeted molecules development. World J Oncol 6: 243-261, 2015. 